\title{
Single Ion Implantation of Bismuth
}

\section{Nathan Cassidy* Paul Blenkinsopp Ian Brown Richard J. Curry Ben Murdin Roger Webb David} Cox*

\section{N. Cassidy}

Ion Beam Centre, Advanced Technology Institute, University of Surrey, Guildford GU2 7XH, UK

Email Address: n.cassidy@surrey.ac.uk

P. Blenkinsopp

Ionoptika Ltd., B6 Millbrook Close, Chandler's Ford, Hampshire, SO534BZ, UK

Dr. I.Brown

Ionoptika Ltd., B6 Millbrook Close, Chandler's Ford, Hampshire, SO534BZ, UK

Prof. R. J. Curry

The Photon Science Institute, Department of Electrical and Electronic Engineering, The University of Manchester, Manchester M13 9PY, UK

Prof. B. N. Murdin

Advanced Technology Institute, University of Surrey, Guildford GU2 7XH, UK

Prof. R. P. Webb

Ion Beam Centre, Advanced Technology Institute, University of Surrey, Guildford GU2 7XH, UK

Dr. D. C. Cox

Advanced Technology Institute, University of Surrey, Guildford GU2 7XH, UK

Email Address: d.cox@surrey.ac.uk

Keywords: quantum technologies, single ion implantation, ion implantation, semiconductor doping, FIB, KeV single ion detection

\begin{abstract}
We present the results from a focused ion beam instrument designed to implant single ions with a view to the fabrication of qubits for quantum technologies. The difficulty of single ion implantation is accurately counting the ion impacts. This has been achieved here through the detection of secondary electrons generated upon each ion impact. We report implantation of single bismuth ions with different charge states into $\mathrm{Si}, \mathrm{Ge}, \mathrm{Cu}$ and $\mathrm{Au}$ substrates, and we determine the counting detection efficiency for single ion implants and the factors which affect such detection efficiencies. We found that for $50 \mathrm{keV}$ implants of $\mathrm{Bi}^{++}$ions into silicon we can achieve a $89 \%$ detection efficiency, the first quantitative detection efficiency measurement for single ion implants into silicon without implanting through a thick $\mathrm{SiO}_{2}$ film. This level of counting accuracy provides implantation of single impurity ions with a success rate significantly exceeding that achievable by random (Poissonian) implantation.
\end{abstract}

\section{Introduction}

Single impurity atoms in semiconductors and insulators are receiving significant interest for quantum technology and other applications. Large arrays of impurity atoms are needed, requiring a repeatable and scaleable method of positioning single atoms with nanometer precision, often referred to as deterministic doping. Single atom lithographic techniques based on scanning probes have managed to achieve the positioning of single dopants $[1,2,3,4]$ atoms with precision of less than a nanometer and high success rates, but the technique is (at present) limited to a small number of species in a small number of substrates, and it is relatively slow. Ion implantation, on the other hand, is much less precise in terms of positioning, but is much more flexible in terms of species and host, and is potentially much faster and easier to scale up for such purposes. Single ion implantation $[5,6,7,8,9]$ has already allowed the manufacture of single phosphorus atom devices in silicon $[10,11,12]$. The main difficulty with ion beam methods is the selection or counting of single ion impacts. Pre-implant methods detect or select an ion before implantation $[13,8,14]$ and they can be extremely accurate but at a cost of very low rate of implant [15]. Post-implant methods use the signals generated from the substrate upon each ion impact. The recorded signal can be the ion beam induced charge (IBIC) [16, 11, 10, 12, 17], which is also very accurate but requires significant pre-processing on-chip. Ion implantation counting in this paper is achieved using a secondary electron detection (SED) method [18, 19, 20, 21, 22, 7, 6, 23, 24, 25]. This method has the advantage of being fast, scalable, and offering freedom in the selection of implant species and target sample, with no pre-processing other than alignment markers. The main challenge with SED is to 
achieve high enough counting accuracy for useful species implanted into useful substrates. It has been discounted by other authors [11] as being much less sensitive than IBIC.

SED methods so far have achieved $90 \%$ detection efficiency for single ion implants into a $25 \mathrm{~nm} \mathrm{SiO}_{2}$ film on $\mathrm{Si}[19,22]$, increasing to almost $100 \%$ by increasing the number of SEs through controlling the substrate bias voltage [23]. Previous work has shown how different target substrates and implant energies result in a different SE yield [26, 27, 28, 29], so here we investigate $\mathrm{Si}$, Ge, $\mathrm{Cu}$ and Au substrates. Silicon was selected as it a promising substrate for quantum technologies as mentioned above, as is germanium [30]. We also used two different implant energies. Previous work suggested that the SED approach might be a viable route for manufacture of single ion devices, but these have not quantified the efficiency for implants into silicon [31]. We are aware of only two reports of a quantified efficiency [19, 23] for which the target was covered in a thick $\mathrm{SiO}_{2}$ film. Implanting through a thick $\mathrm{SiO}_{2}$ film has two disadvantages: the method increases the likelihood of scattering of oxygen atoms from the film into the silicon matrix during impact, introducing contaminant spins which could ruin coherence times of any donor-based qubit; and the ion can also be stopped in the over-layer. In this work we report the first quantitative study for the detection efficiency for single ion implants into silicon using the SED approach. As we shall show, the efficiency can be very high.

Because of the availability of much more convenient alternatives (phosphorus and arsenic), bismuth is not used as a conventional dopant by the semiconductor industry. However neutral bismuth donors have shown excellent potential as a spin qubit $[32,33,34]$ thanks to large nuclear spin, and a strong hyperfine coupling constant (compared with $\mathrm{P}$ and As) and respectably long electron spin coherence times [35, 36]. The downside of implanting heavy mass ions such as bismuth is that the target silicon lattice will incur a large amount of damage during the implantation process, which along with the low solubility of bismuth in silicon [37] requires careful selection of annealing times and temperatures [38].

A limiting factor for ion implantation in some applications is the ion straggle, though in some quantum computing designs [39] the impurity spacing and tolerance are quite large (400nm and 12nm respectively). Even if the spot-size of a focused ion beam is $10 \mathrm{~nm}$, when phosphorus ion is implanted into silicon at $25 \mathrm{keV}$ the ion range is $38 \mathrm{~nm}$ and the lateral straggle is $17 \mathrm{~nm}$ - very close to the desired tolerance (all ion stopping values calculated using SRIM [40]). The ion straggle is inversely proportional to the ion mass, and bismuth has the largest atomic mass of all group V silicon donors. Bismuth implanted at 25 $\mathrm{keV}$ into silicon has an ion range of $20 \mathrm{~nm}$ and a lateral straggle of $4 \mathrm{~nm}$, so there is a great advantage in spatial precision when implanting this particular element (this has already led to it being selected for some single atom devices [41]).

\section{The SIMPLE tool}

In this paper we demonstrate the deterministic implantation of single bismuth ions in silicon, using a focused ion beam (FIB) tool named the Single Ion Multi-species Positioning at Low Energy (SIMPLE) tool, manufactured by Ionoptika Ltd. The tool has a $25 \mathrm{kV}$ accelerating anode, and uses a liquid metal ion source (LMIS) along with electrostatic lenses to achieve a beam spot size of $15 \pm 2 \mathrm{~nm}$. This spotsize was obtained using the rise distance method in which a beam is swept across a sharp discontinuity ("knife-edge"). The method and its limitations are detailed in [42]. Deterministic single ion implantation is achieved through the collection of SEs upon ion impact using DeTech XP-2352 channel electron multipliers placed $5 \mathrm{~mm}$ away from implant site (seen in Figure 3) with a front end voltage of $500 \mathrm{~V}$. Any species that can be incorporated into a LMIS can be implanted. A list of possible species can be found in Ref [43]. The tool has the imaging capabilities of a standard FIB, meaning one can use an electron beam lithography-like system for computer-based pattern design and alignment to existing features (e.g a Nabity system https://www.jcnabity.com/). The alignment means as well as having a spatial resolution of sub-20 nm for ion positioning, the tool can target sub- $\mu \mathrm{m}$ regions for implantation e.g a MOSFET channel region. 
The LMIS used in this work produces many different bismuth cations and poly-atomic ion species which can be selected using a Wien filter so that the tool only implants one particular species with a specific charge state i.e $\mathrm{Bi}^{++}$. The extraction of single ions is achieved through fast ion beam blanking whilst utilising low beam currents. The current produced by the SIMPLE tool can be controlled by attenuating the beam using a series of apertures sized from $300 \mu \mathrm{m}$ to $10 \mu \mathrm{m}$.

\section{Method}

\section{Single Ion Implantation}

The average number of ions per pulse, $\lambda$ is a product of the beam current, $I$ and the pulse length, $t$

$$
\lambda=\frac{I t}{C q}=L t
$$

where $L$ is the number of ions per unit time, and $C q$ is the charge of the ion, i.e. the factor $C$ is the charge state of the ion species. For example, in the case of a beam of $\mathrm{Bi}^{++}$ions, $C=2$. The number of ions in a pulse (and therefore extracted by the ion gun), $X$, follows a Poisson distribution with mean $\lambda$. Supposing we aim to have one ion implanted with each pulse, then the probability of an error-free implant $P(X=1)=\lambda e^{-\lambda}$. This has an optimum of $37 \%$ which occurs when $\lambda=1$. In this case the probability that the pulse has no ion is $P(X=0)=e^{-\lambda}=37 \%$ also, and therefore there is a $26 \%$ probability of obtaining 2 or more ions.

If implants can be detected efficiently, i.e. without false negatives, then pulses containing no ions can easily be remedied by pulsing again. However errors due to multiple ions in the pulse cannot be corrected and must be eliminated by reducing $\lambda$ as much as possible. For example, if only 1 pulse out of 20 contains an ion, i.e. the average number of ions per pulse is $\lambda=0.05$, there is a $P(X=0)=95.1 \%$ probability of the pulse containing no ion, so the beam must be pulsed many times before an ion implantation event occurs. The advantage is that the probability of the pulse containing more than one ion (the only source of error for perfect detection) drops to only $P(X>1)=1-(1+\lambda) e^{-\lambda}=0.1 \%$. This error probability drops monotonically with $\lambda$, but the improvement is moderated (or even lost) if the detection is imperfect. Thus efficient ion detection is the key to deterministic implantation. False positives (dark counts) must also be considered; whereas false negative events (undetected implants) introduce errors where an implant contains multiple ions, false positives produce implants without ions.

\section{Secondary Electron Detection Efficiency}

Let the SE detection efficiency $\eta$ be the likelihood of detecting a single ion implantation event, in which case $1-\eta$ is the false negative detection rate. The value of $\eta$ depends on the efficiency of collection of SE's, which is instrument dependent, but is also determined by the number of SE's generated per impact. The efficiency is therefore dependent on the target substrate, the ion species and the implant energy. In this work we investigate the implant efficiency for a variety of substrates and energies, for one species $(\mathrm{Bi})$.

If the false positive rate is $K$ (the number of dark counts per second) then the number of dark counts per pulse is $\kappa=K t$. The total number of events per unit time seen by the detector is therefore $N=$ $K+\eta L$ and the total number of events per pulse is $\nu=N t=\kappa+\eta \lambda$. Assuming each dark count event and each ion detection event is independent of any other event, then detected events are governed by a Poisson distribution with mean $\nu$.

There are two ways to find $\nu$ experimentally (and hence obtain $\eta$ ). We could attempt to count the number of detected events, either in one long pulse or averaging over many short pulses, to find $\nu$ directly. It is convenient instead to record experimentally the proportion of pulses that appear empty. In this scenario, we do not attempt to count the number of impacts within any pulse that contains one or more 
Table 1: SE detection efficiency, $\eta=(N-K) / L$, for bismuth ion implants. The total number of events per unit time, $N$ determined from Figs $1 \& 2$ and the ion current $L$ are also given. The dark count $K$ was negligible for this experiment.

\begin{tabular}{lllll}
\hline Target Substrate & Incident Energy $(\mathrm{keV})$ & $L($ ions per $\mu \mathrm{s})$ & $N($ counts per $\mu \mathrm{s})$ & $\eta(\%)$ \\
\hline Silicon & 25 & $0.312 \pm 0.010$ & $0.241 \pm 0.005$ & $78 \pm 3$ \\
Silicon & 25 & $0.296 \pm 0.010$ & $0.238 \pm 0.003$ & $80 \pm 3$ \\
Silicon & 50 & $0.091 \pm 0.005$ & $0.081 \pm 0.001$ & $89 \pm 5$ \\
Germanium & 25 & $0.296 \pm 0.010$ & $0.258 \pm 0.003$ & $87 \pm 3$ \\
Copper & 25 & $0.561 \pm 0.010$ & $0.494 \pm 0.006$ & $88 \pm 2$ \\
Gold & 25 & $0.561 \pm 0.010$ & $0.533 \pm 0.007$ & $95 \pm 2$ \\
\hline
\end{tabular}

events, we only record that it was not empty. In this way we do not need to take account of the deadtime of the detector or its speed of response, so long as the time between pulses is long. The probability of an apparently empty pulse is

$$
p=e^{-\nu}=e^{-N t}=e^{-\kappa-\eta \lambda}
$$

In experiments with fixed pulse duration and varying beam current the last form in Eqn 2 is most useful because the number of dark counts per pulse $\kappa$ is fixed. In our experiment, we varied the value $\lambda$ by varying $t$ for fixed ion current $L$, in which case the penultimate form of Eqn 2 is most useful because $N$ is fixed. For each pulse duration we performed a large number of pulses, $M$ and recorded the number of apparently empty pulses where the detector did not trigger, $m$. So long as $m$ is large, $m / M \rightarrow p$. Eqn 2 leads to the experimental average number of events per pulse, $\nu=-\ln (\mathrm{m} / M)$, and the slope of a plot of the experimental $\nu$ vs the experimental pulse duration $t$ is equal to $N$. Since $m$ is governed by a binomial distribution, the uncertainty in $p$ for finite $m$ is $s(p)=\sqrt{p(1-p) / M}$, and hence the vertical error bars in $\nu$ are

$$
s(\nu)=\sqrt{\left(e^{\nu}-1\right) / M}
$$

Clearly these error bars are not constant, they increase with $\nu$, and ordinary least-squares fitting is inappropriate. Linear regression fitting of $\nu=N t$ to the experimental $\nu(t)$, taking this error variation $s(\nu)$ into account, produces the best fit value of $N$ and its error, which is

$$
N=\frac{\mathcal{S}(\nu t)}{\mathcal{S}\left(t^{2}\right)} \pm \frac{1}{\sqrt{ } \mathcal{S}\left(t^{2}\right)}
$$

where $\mathcal{S}(u)=\sum_{i} u_{i} / s_{i}^{2}$ and $\nu_{i}$ is the experimental value of $-\ln \left(m_{i} / M\right)$ when the pulse duration was $t_{i}$, and $s_{i}=s\left(\nu_{i}\right)$ from Eqn 3 is the corresponding error bar. From the value of $N$ we can extract $\eta$ so long as the dark count rate and ion current $(K$ and $L)$ are known.

\section{Experiment and Results}

The silicon and germanium surfaces were prepared by an oxygen plasma ash (100 W for 5 min) to remove any organic matter. All target substrates were then rinsed with acetone, IPA and methanol. In the transfer of the sample to the chamber some native oxide will form. It has been well documented that the native oxide formed on silicon is limited to a few $\mathrm{nm}$ [44]; experiments have shown freshly etched silicon to have a growth of oxide to be $1 \mathrm{~nm}$ thick after a few hours, and remaining at that thickness after 24 hours exposure to air at $24^{\circ} \mathrm{C}[45]$.

We measured the dark count rate $K$ detected by the SE detector by blanking the beam and looking for events. In several experiments with a total integration time of 0.1 sec the number of dark count events detections was about 1 , i.e. $K \approx 10^{-5}$ counts per $\mu$ s. This is many orders of magnitude less than any of the ion flow rates used in the experiment, i.e. $K \ll N$ and the effect of dark counts can be neglected. Obviously a careful measurement of $L$ is still crucial in order to extract $\eta=N / L$.

The beam current produced by the SIMPLE tool is measured using a Keithley picoammeter connected to a Faraday cup on the sample stage. A typical current used for this work was $I=45 \mathrm{fA}$, which corre- 


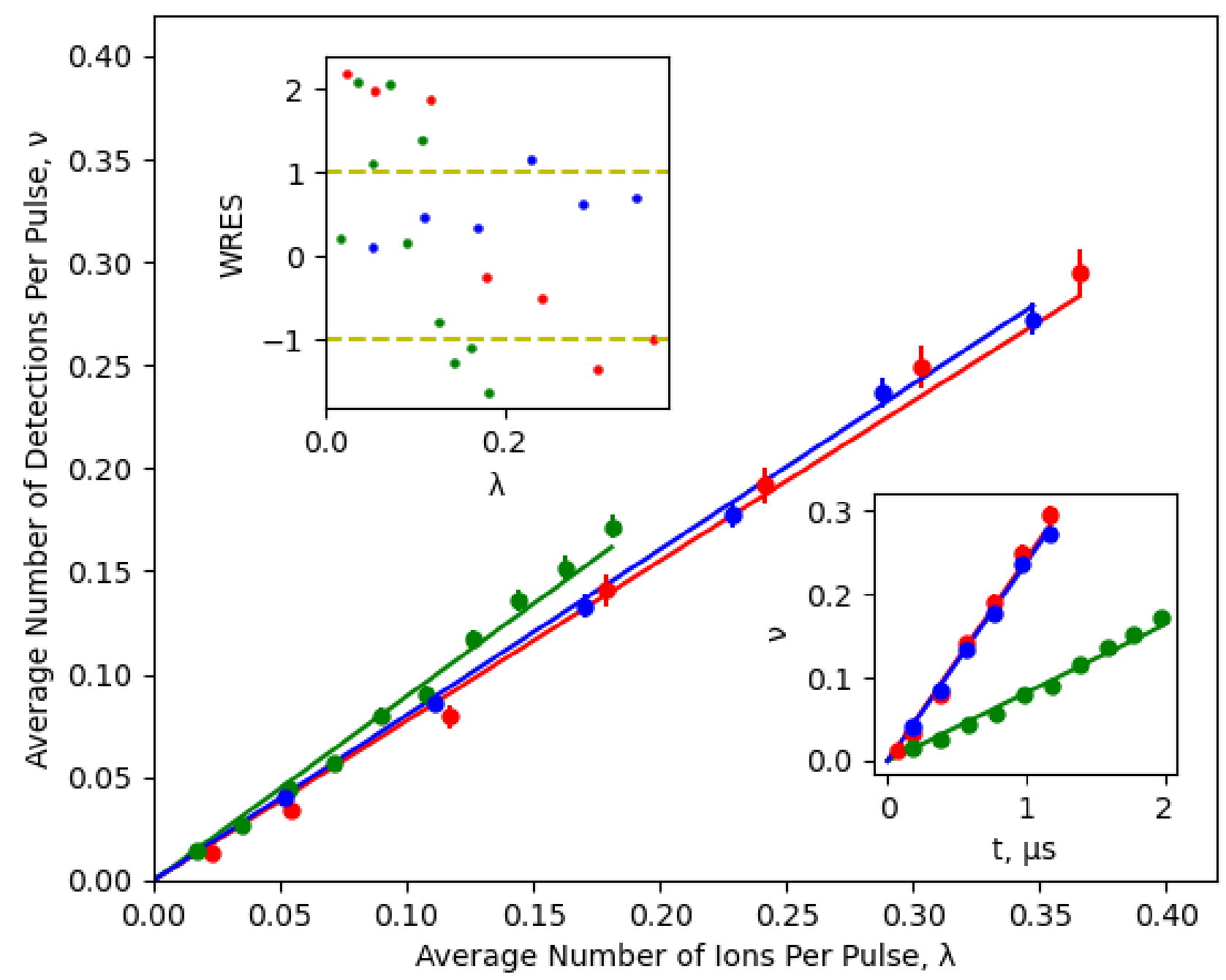

$25 \mathrm{keV} \mathrm{Bi}^{+} \mathrm{I}=50 \mathrm{~A}$

$50 \mathrm{keV} \mathrm{Bi}^{++}$

$25 \mathrm{keV} \mathrm{Bi}^{+} \mathrm{I}=48 \mathrm{fA}$

Figure 1: Detection efficiency calibration for $\mathrm{Bi}^{++}$ions implanted with $50 \mathrm{keV}$, and $\mathrm{Bi}^{+}$ions implanted with $25 \mathrm{keV}$, both into silicon. The symbols show the average number of detected events per pulse, taken from the measured empty pulse fraction $\nu=-\ln p=-\ln (m / M)$ for fixed current values $I=29 \mathrm{fA}$ and $I=50 \mathrm{fA}$ for the $\mathrm{Bi}^{++}$and $\mathrm{Bi}^{+}$beams respectively (corresponding to $L=0.09$ and $L=0.32$ ions per pulse) and varying $t$. The lower right inset shows $\nu(t)$ with linear regression fits as described by Eqn 4 for the slope, $N$, the total detected event rate (and its error). With the value of $N$ obtained, the fit line $\nu=N t$ can be converted to $\nu=N \lambda / L=\eta \lambda$ (since the dark count rate is negligible in this experiment $K \ll N$ ), shown in the main figure, in which the slope is obviously $\eta$. The weighted residuals from the fit are shown in the upper right inset. Values of the detection efficiency $\eta=N / L$ are given in Table 1. 


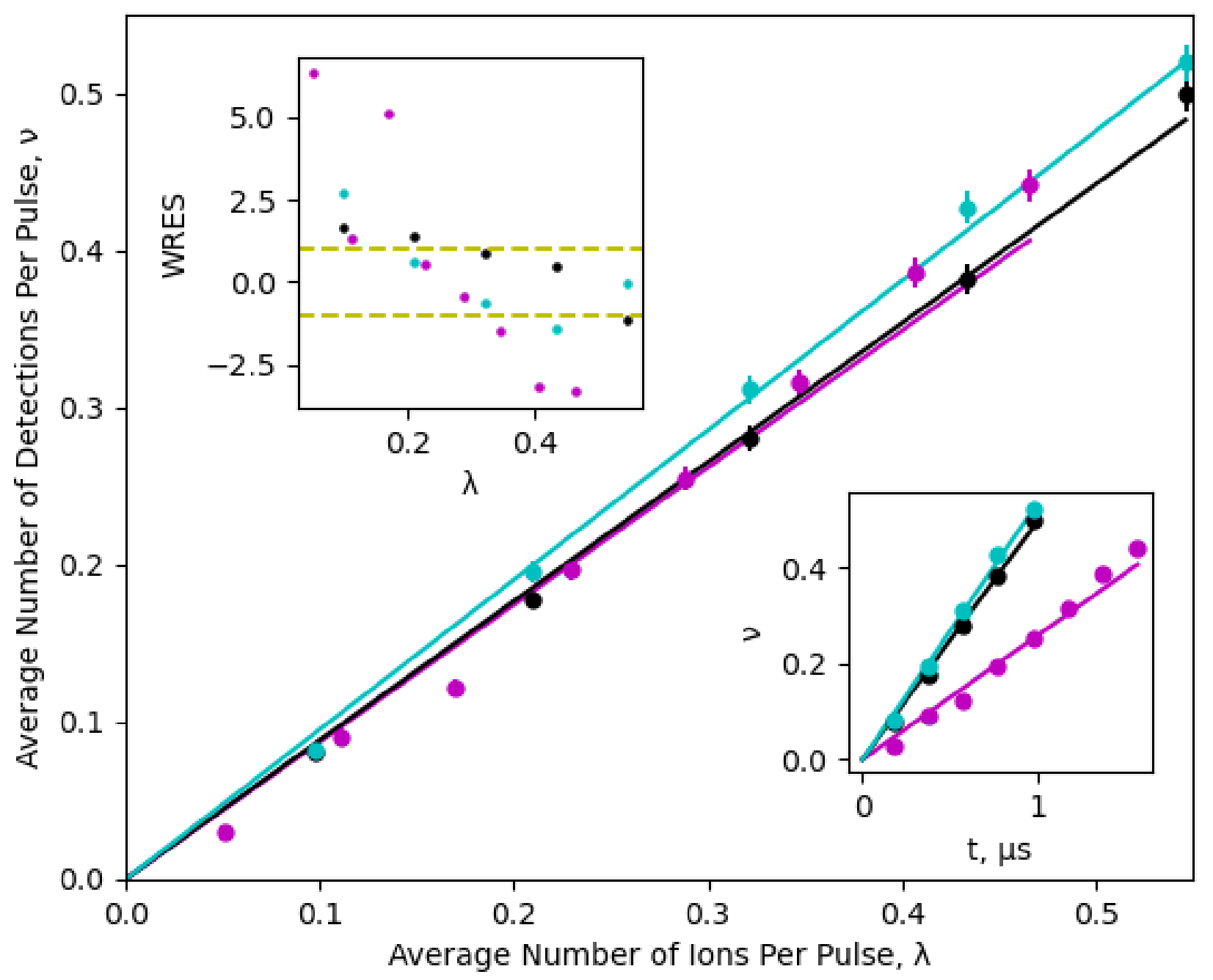

\$ Germanium $\quad$ Copper $\quad$ Gold

Figure 2: Detection efficiency calibration for $\mathrm{Bi}^{+}$ions implanted with $25 \mathrm{keV}$ into silicon, gold, copper and germanium substrates. In the experiment the ion current was measured to be $I=90 \mathrm{fA}$ for the implants into gold and copper, and $I=48 \mathrm{fA}$ for implants into germanium (corresponding to values of $L=0.56$ and $L=0.29$ respectively). The slope, $N$, was found in the same way as for Figure 1, and the inferred value of $\eta=N / L$ is given in Table 1. 


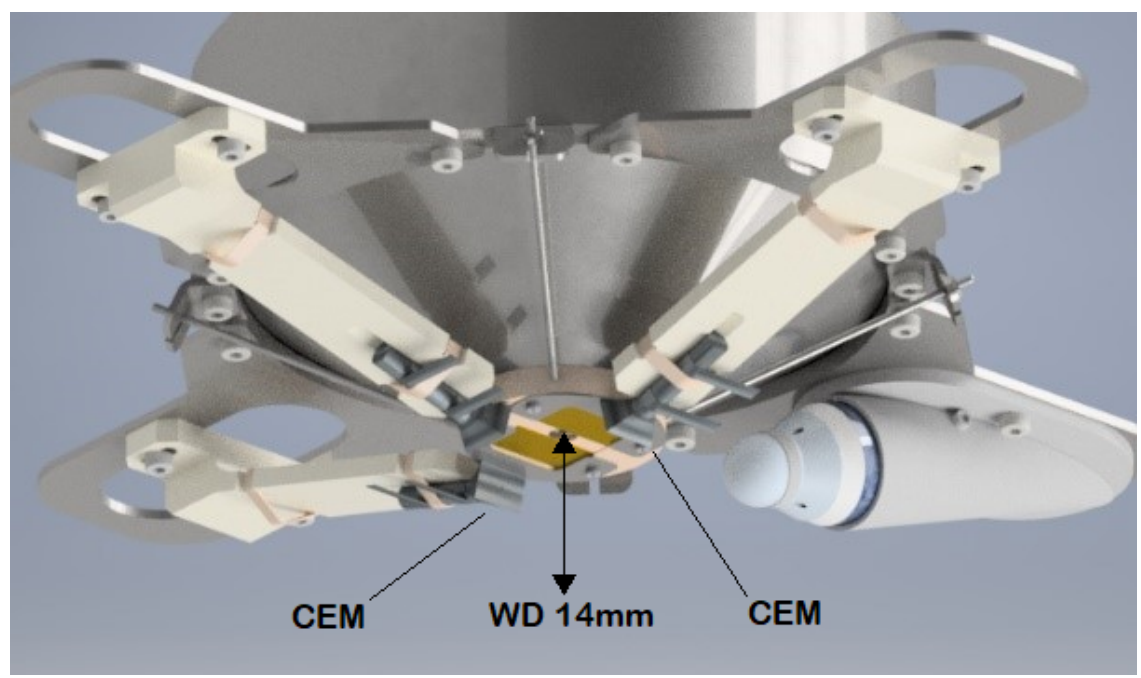

Figure 3: Ionoptika Ltd. detector array on the SIMPLE tool. Labelled are the channel electron multipliers (CEM) which collect the SE signal. These are positioned on the nose cone of the ion gun $10 \mathrm{~mm}$ distance from the implant site. The working distance of the tool is $14 \mathrm{~mm}$.

sponds to an ion current of $L=0.28$ ions $/ \mu$ s for $\mathrm{Bi}^{+}$or 0.14 ions $/ \mu \mathrm{s}$ for $\mathrm{Bi}^{++}$ions. The LMIS is a dynamic system, which means the beam current can drift over time, typically varying by $\pm 1 \mathrm{fA}$ across an hour of LMIS use. Occasionally emission from the LMIS became unstable due to collapse of the Taylor cone $[46,47]$ with the sample current fluctuating on and off. In such cases data was discarded.

The pulse duration was controlled by a pair of electrostatic deflection plates. The time taken for an ion to traverse these blanking plates reduces the duration over which the gate is open relative to the duration of the voltage applied to them by an amount $T_{0}$, and this was calculated to be $26 \mathrm{~ns}$ for a $25 \mathrm{keV}$ bismuth ion and $19 \mathrm{~ns}$ for a $50 \mathrm{keV}$ ion. In order to confirm this and to obtain measurements for the errors in both the pulse duration and ion current, we positioned a channel electron multiplier (same detector as used for the SE measurements) at the sample position and obtained the measured number of events per pulse $\nu$ from the proportion of empty pulses as described in the previous section. We varied the nominal pulse duration $T$ and performed a linear regression for $\nu=L\left(T-T_{0}\right)$, taking into account the systematic variation in the error bars $s(\nu)$ given by Eqn 3. In this case $L=a / b \pm \sqrt{ }(\mathcal{S}(1) / b)$ and $T_{0}=c / a \pm \sqrt{ }\left(\mathcal{S}\left(t^{2}\right) b / a^{2}\right)$ where $a=\mathcal{S}(1) \mathcal{S}(\nu t)-\mathcal{S}(\nu) \mathcal{S}(t), b=\mathcal{S}(1) \mathcal{S}\left(t^{2}\right)-\mathcal{S}(t)^{2}, c=\mathcal{S}(t) \mathcal{S}(\nu t)-\mathcal{S}(\nu) \mathcal{S}\left(t^{2}\right)$. We found a slope of $L=0.271 \pm 0.002$ ions per $\mu$ s for a nominal ion current of $L=0.28$ ions per $\mu$ s taken from the Faraday cup, so the discrepancy gives us the scale of the uncertainty in the Faraday cup measurement, which is therefore 0.01 ions per $\mu \mathrm{s}$. From the fit we obtain $T_{0}=26 \pm 2 \mathrm{~ns}$, which is in excellent agreement with the calculated traverse time. The actual pulse duration $t=T-T_{0}$ used for all measurements described below was therefore given by the nominal pulse duration minus 26 ns for 25 $\mathrm{keV}$ ions and minus $19 \mathrm{~ns}$ for $50 \mathrm{keV}$ ions. This benchmark also gives us an upper limit on the error in the pulse duration of $2 \mathrm{~ns}$, which is proportionally much smaller than any of the the values for fractional errors in $L$, or any of the values of the fractional binomial errors in $\nu$.

By changing the gun voltage and using the Wien filter to select various ion charge states, we can change the energy of the implantation. The maximum anode of the gun is $25 \mathrm{kV}$, at these settings the $\mathrm{Bi}^{+}$charge state implants with $25 \mathrm{keV}$ of energy, and the $\mathrm{Bi}^{++}$ion implants with $50 \mathrm{keV}$ of energy. We investigated the efficiency with which $\mathrm{Bi}^{+}$and $\mathrm{Bi}^{++}$impacts into $\mathrm{Si}$ are counted by the $\mathrm{SE}$ detector, Fig 1 . For these tests we fixed the beam current and varied the pulse length, $t$, between 0.1 and $1.2 \mu \mathrm{s}$. The empty pulse count $m$ was obtained for $M=5,000$ pulses for a fixed value of $L$ and converted to an average number of events per pulse $\nu$ using Eqn 2, shown as insets in Figure 1. The value of $N$ (with its error) was obtained from $\nu(t)$ using Eqn 4, from which we obtain the detection efficiency $\eta$ using the known $L$. In this measurement the fractional error in $L(4 \%)$ is significantly larger than the fractional error in $N$ (typically $1 \%$ ), and therefore the error in $\eta$ is also $4 \%$, dominated by the error in the ion current. Convert- 
ing $t$ to $\lambda$ using the same $L$ allows us to plot $\nu(\lambda)$ whose slope is $\eta$. A similar analysis was performed for $\mathrm{Bi}^{+}$implants into a variety of other substrates shown in Fig 2. Table 1 summarises the SE detection efficiency results.

The weighted residuals from the fits of $\nu=N t$, i.e. $\left(N t_{i}-\nu_{i}\right) / s_{i}$ are shown against $\lambda_{i}=L t_{i}$ as insets in Figs 1 and 2. It is to be expected that the residuals show Gaussian scatter about zero with an s.d. of 1 . Although the scatter is close to expectation, drift of the LMIS appears to have led to systematically increasing $N$ as $t$ increases. In a proportional fit fixed at the origin, the points at large $t$ have the biggest contribution (in spite of their larger error), so it is to be expected that the residuals are scattered about zero at high $t$, and the low- $t$ residuals are automatically the ones that appear scattered about a non-zero mean. Since the data were taken in order of increasing $t_{i}$, upward drift in $N$ with $t_{i}$ means $L$ drifted upwards from the point that it was measured at the start of the experiment. It takes about one hour to collect all the points. We do not believe this drift has influenced our results significantly because the contribution to the error in our quoted results for $\eta$ due to the drift is less than the contribution from the error in $L$.

\section{Discussion}

There are two mechanisms for SE emission, kinetic electron emission (KEE) and potential electron emission, and there is a threshold for the incoming ion velocity above which the former dominates [48]. In this work the incoming bismuth ion velocity is above the KEE velocity threshold for all target substrates and ion energies. In the KEE mechanism, the incident ion loses energy throughout the target substrate due to elastic and inelastic collisions in the solid target. The bismuth ions used here are heavy projectiles, so energy is transferred to the electrons from direct binary collisions between the ion and nearly free valence-band electrons [48, 49]. Therefore when comparing the detection efficiency for each incident energy and target material, the main factors affecting the SE yield (and therefore detection efficiency) are the energy deposition and the electron mean free path in the material [50].

The observed increase in detection efficiency for increasing energy of the incident ions seen in Figure 1 matches previous experiments and observations that at velocities above the KEE threshold, the SE yield increases with increasing ion energy [50]. Using Stopping and Range of Ions in Matter (SRIM) modelling software [40] we found that $50 \mathrm{keV}$ ions implanted into Si produce 43 ionization events per ion compared to $25 \mathrm{keV}$ ions which produce 40 ionization events per ion. This small increase in ionization number explains when there is only a small increase in the detection efficiency.

Figure 2 shows the efficiency plot for the same conditions as Figure 1 using the same Bi ion doses at 25 $\mathrm{keV}$ of energy, for implants into gold, copper and germanium substrates. We have generally observed that the higher atomic mass substrates result in higher detection efficiencies for $25 \mathrm{keV} \mathrm{Bi}^{+}$ions. The rise in SE yield with target substrate atomic mass is not monotonic - the KEE yield is complicated by the bulk surface contributions [29]. The electronic energy deposition per nm of depth was calculated using the SUSPRE ion implantation calculator [51]. For incident $25 \mathrm{keV}$ Bi ions the energy deposition for the targets used was as follows: $\mathrm{Si}, 180 \mathrm{eV}$; Cu, $346 \mathrm{eV}$; Ge, $211 \mathrm{eV}$; Au, $452 \mathrm{eV}$. The SE yield increases with increasing energy deposition and increasing probability of electron escape - the latter scaling with the electron mean free path. The energy deposition is greater for gold than for copper, explaining why the SE yield is likely greater for gold - resulting in a higher detection efficiency. The same is true for germanium when compared to silicon. The detection efficiency for implants into germanium was very similar to that for copper. In spite of the far greater energy deposition for metals than for semiconductors, more electrons are able to escape from the surface of the germanium substrate because the electron mean free path is greater for insulators [50].

Any effect of the unintentional native oxide on the silicon targets in this work may be ignored. Previous work on deterministic single ion implantation has obtained very high detection efficiencies by implanting through a thick $(30 \mathrm{~nm}) \mathrm{SiO}_{2}$ film. In the case of implanting through a thin native $\mathrm{SiO}_{2}$ film of $2 \mathrm{~nm}$ 
results in almost all of the energy being deposited in the silicon and the electron yield matches that obtained from clean silicon surfaces [27].

\section{Conclusions}

We now can deterministically implant single bismuth ions into silicon with $89 \%$ probability. We are not aware of any other quantitative values for efficiency for detection of implants into silicon using secondary electron detection. This approaches truly deterministic implantation and will open the possibility of producing very large arrays of single dopant atoms with minimal errors. For context, the optimum probability to produce such an array without impact counting determinism (i.e. using one pulse for each spot with $\lambda=1$ ) is $0.01 \%$. Ignoring the issue of activation post- implantation, the current level of SE detection performance gives a $35 \%$ probability of correctly placing all 9 single ions in a $3 \times 3$ array without error. This success probability could be increased with, for example, optimised with sample biasing [23], which is the subject of on-going research.

\section{Acknowledgements}

The authors are grateful to the UK-EPSRC through SIMPLE (EPSRC grant ref EP/N015215) and ADDRFSS (EPSRC grant ref EP/M009564). NC is grateful to the EPSRC CDT in Micro- and Nanomaterials (EPSRC grant ref EP/L016788).

\section{References}

[1] M. Fuechsle, J. A. Miwa, S. Mahapatra, H. Ryu, S. Lee, O. Warschkow, L. C. L. Hollenberg, G. Klimeck, M. Y. Simmons, Nature nanotechnology 2012, 7, 4242.

[2] B. Voisin, B. Roche, E. Dupont-Ferrier, B. Sklenard, M. Cobian, X. Jehl, O. Cueto, R. Wacquez, M. Vinet, Y.-M. Niquet, S. De Franceschi, M. Sanquer, In 2013 Proceedings of the European SolidState Device Research Conference (ESSDERC). IEEE, ISBN 978-1-4799-0649-9, 2013 147-150.

[3] T. J. Z. Stock, O. Warschkow, P. C. Constantinou, J. Li, S. Fearn, E. Crane, E. V. S. Hofmann, A. Kölker, D. R. Mckenzie, S. R. Schofield, N. J. Curson, C. Authors, Atomic-Scale Patterning of Arsenic in Silicon by Scanning Tunneling Microscopy, Technical report.

[4] P. M. Koenraad, M. E. Flatté 2011.

[5] D. N. Jamieson, S. Prawer, I. Andrienko, D. A. Brett, V. Millar, Nuclear Instruments and Methods in Physics Research Section B: Beam Interactions with Materials and Atoms 2001, 175-177 744.

[6] T. Shinada, S. Okamoto, T. Kobayashi, I. Ohdomari, Nature 2005, 437, 70621128.

[7] T. Schenkel, A. Persaud, S. J. Park, J. Meijer, J. R. Kingsley, J. W. McDonald, J. P. Holder, J. Bokor, D. H. Schneider, Journal of Vacuum Science and Technology B: Microelectronics and Nanometer Structures 2002, 20, 62819.

[8] J. Meijer, T. Vogel, B. Burchard, I. Rangelow, L. Bischoff, J. Wrachtrup, M. Domhan, F. Jelezko, W. Schnitzler, S. Schulz, K. Singer, F. Schmidt-Kaler, Applied Physics A 2006, 83, 2321.

[9] D. N. Jamieson, W. I. Lawrie, S. G. Robson, A. M. Jakob, B. C. Johnson, J. C. McCallum, Materials Science in Semiconductor Processing 2017, 62 23.

[10] J. van Donkelaar, C. Yang, A. D. C. Alves, J. C. McCallum, C. Hougaard, B. C. Johnson, F. E. Hudson, A. S. Dzurak, A. Morello, D. Spemann, D. N. Jamieson, Journal of Physics: Condensed Matter 2015, 27, 15154204. 
[11] D. N. Jamieson, W. I. Lawrie, S. G. Robson, A. M. Jakob, B. C. Johnson, J. C. McCallum, F. E. Hudson, A. S. Dzurak, A. Morello, In Proceedings of the International Conference on Ion Implantation Technology. Institute of Electrical and Electronics Engineers Inc., ISBN 9781509020232, 2017

[12] C. Yang, D. N. Jamieson, C. Pakes, S. Prawer, A. Dzurak, F. Stanley, P. Spizziri, L. Macks, E. Gauja, R. G. Clark, In Japanese Journal of Applied Physics, Part 1: Regular Papers and Short Notes and Review Papers, volume 42, ISSN 00214922, 2003 4124-4128.

[13] G. Jacob, K. Groot-Berning, S. Wolf, S. Ulm, L. Couturier, S. T. Dawkins, U. G. Poschinger, F. Schmidt-Kaler, K. Singer 2016.

[14] P. Räcke, D. Spemann, J. W. Gerlach, B. Rauschenbach, J. Meijer, Scientific Reports 2018, 8, 1 9781.

[15] K. Groot-Berning, T. Kornher, G. Jacob, F. Stopp, S. T. Dawkins, R. Kolesov, J. Wrachtrup, K. Singer, F. Schmidt-Kaler 2019.

[16] D. N. Jamieson, C. Yang, T. Hopf, S. M. Hearne, C. I. Pakes, S. Prawer, M. Mitic, E. Gauja, S. E. Andresen, F. E. Hudson, A. S. Dzurak, R. G. Clark, Applied Physics Letters 2005, 86, 201.

[17] J. L. Pacheco, M. Singh, D. L. Perry, J. R. Wendt, G. Ten Eyck, R. P. Manginell, T. Pluym, D. R. Luhman, M. P. Lilly, M. S. Carroll, E. Bielejec, Rev. Sci. Instrum 2017, 88123301.

[18] T. Matsukawa, T. Fukai, S. Suzuki, K. Hara, M. Koh, I. Ohdomari, Applied Surface Science 1997, $117-118677$.

[19] T. Shinada, Journal of Vacuum Science Technology B: Microelectronics and Nanometer Structures 1998, 16, 42489.

[20] T. Matsukawa, T. Shinada, T. Fukai, I. Ohdomari, Journal of Vacuum Science Technology B: Microelectronics and Nanometer Structures 1998, 16, 42479.

[21] T. Shinada, A. Ishikawa, M. Fujita, K. Yamashita, I. Ohdomari, Japanese Journal of Applied Physics 1999, 38, Part 1, No. 6A 3419.

[22] T. Shinada, A. Ishikawa, C. Hinoshita, M. Koh, I. Ohdomari, Applied Surface Science 2000, 162 499.

[23] T. Shinada, T. Kurosawa, H. Nakayama, Y. Zhu, M. Hori, I. Ohdomari, Nanotechnology 2008, 19, 34345202 .

[24] T. Shinada, M. Hori, Y. Ono, K. Taira, A. Komatsubara, T. Tanii, T. Endoh, I. Ohdomari, In Alternative Lithographic Technologies II, volume 7637. 2010763711.

[25] M. Hori, T. Shinada, K. Taira, A. Komatsubara, Y. Ono, T. Tanii, T. Endoh, I. Ohdomari, Applied Physics Express 2011, 4, 446501.

[26] S. Ullah, A. H. Dogar, A. Qayyum, Nuclear Instruments and Methods in Physics Research, Section B: Beam Interactions with Materials and Atoms 2009, 267, 183059.

[27] H. Jacobsson, G. Holmén, Physical Review B 1994, 49, 31789.

[28] K. Wittmaack, Nuclear Inst. and Methods in Physics Research, B 1991, 58, 3-4 317.

[29] K. Ohya, T. Ishitani, Target material dependence of secondary electron images induced by focused ion beams, Technical report, 2002.

[30] G. Scappucci, G. Capellini, B. Johnston, W. M. Klesse, J. A. Miwa, M. Y. Simmons, Nano Letters 2011, 11, 62272 . 
[31] T. Schenkel, C. D. Weis, C. C. Lo, A. Persaud, I. Chakarov, D. H. Schneider, J. Bokor, In AIP Conference Proceedings, volume 1640. American Institute of Physics Inc., ISBN 9780735412798, ISSN 15517616, 2015 124-128.

[32] C. D. Weis, C. C. Lo, V. Lang, A. M. Tyryshkin, R. E. George, K. M. Yu, J. Bokor, S. A. Lyon, J. J. Morton, T. Schenkel, Applied Physics Letters 2012, 100, 17172104.

[33] M. H. Mohammady, G. W. Morley, A. Nazir, T. S. Monteiro, Physical Review B - Condensed Matter and Materials Physics 2012, 85, 9094404.

[34] G. Wolfowicz, A. M. Tyryshkin, R. E. George, H. Riemann, N. V. Abrosimov, P. Becker, H. J. Pohl, M. L. Thewalt, S. A. Lyon, J. J. Morton, Nature Nanotechnology 2013, 8, 8561.

[35] R. E. George, W. Witzel, H. Riemann, N. V. Abrosimov, N. Nötzel, M. L. Thewalt, J. J. Morton, Physical Review Letters 2010, 105, 6.

[36] G. W. Morley, M. Warner, A. M. Stoneham, P. T. Greenland, J. Van Tol, C. W. M. Kay, G. Aeppli, Nature Materials 2010.

[37] F. A. Trumbore, Bell System Technical Journal 1960, 39, 1205.

[38] T. Peach, K. Homewood, M. Lourenco, M. Hughes, K. Saeedi, N. Stavrias, J. Li, S. Chick, B. Murdin, S. Clowes, Advanced Quantum Technologies 2018, 1, 21870021.

[39] J. O'gorman, N. H. Nickerson, P. Ross, J. J. Morton, S. C. Benjamin, npj Quantum Information 2016, 2, 11 .

[40] J. F. Ziegler, M. Ziegler, J. Biersack, Nuclear Instruments and Methods in Physics Research Section B: Beam Interactions with Materials and Atoms 2010, 268, 11-12 1818.

[41] A. Persaud, J. A. Liddle, T. Schenkel, J. Bokor, T. Ivanov, I. W. Rangelow, Journal of Vacuum Science and Technology B: Microelectronics and Nanometer Structures 2005, 23, 62798.

[42] J. Orloff, In Scanning Microscopy 2010, volume 7729. SPIE, 2010 77290C.

[43] L. Bischoff, P. Mazarov, L. Bruchhaus, J. Gierak, Applied Physics Reviews 2016, 3, 2021101.

[44] C. Bohling, W. Sigmund, Silicon 2016, 8339.

[45] S. I. Raider, Journal of The Electrochemical Society 1975, 122, 3413.

[46] E. Hesse, W. Driesel, C. Dietzsch, L. Bischoff, J. Teichert, Japanese Journal of Applied Physics 1996, 35, Part 1, No. 105564.

[47] W. Driesel, C. Dietzsch, R. Mühle, Citation: Journal of Vacuum Science Technology B: Microelectronics and Nanometer Structures Processing 1996, 143367.

[48] R. A. Baragiola, E. V. Alonso, J. Ferron, A. Oliva-Florio, Surface Science 1979, 90, 2240.

[49] J. Ferron, E. V. Alonso, R. A. Baragiola, A. Oliva-Florio, Journal of Physics D: Applied Physics 1981, 14, 91707.

[50] D. Hasselkamp, 1-95. Springer, Berlin, Heidelberg, 1992.

[51] R. P. Webb, I. Wilson, Simulation of Semiconductor Devices and Processes 1986, 2247. 